\title{
Height Dependence of the Penumbral Fine-scale Structure in the Inner Solar Atmosphere
}

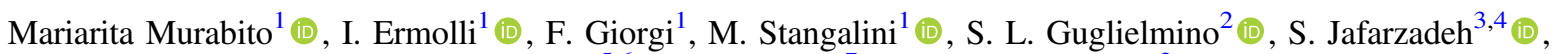 \\ H. Socas-Navarro ${ }^{5,6}$ (iD, P. Romano ${ }^{7}$ (D) and F. Zuccarello ${ }^{2}$ (i) \\ ${ }_{1}^{1}$ INAF-Osservatorio Astronomico di Roma, Via Frascati, 33, I-00078 Monte Porzio Catone, Italy; mariarita.murabito@inaf.it \\ ${ }^{2}$ Dipartimento di Fisica e Astronomia-Sezione Astrofisica, Universitàdegli Studi di Catania, Via S. Sofia 78, I-95123 Catania, Italy \\ ${ }^{3}$ Rosseland Centre for Solar Physics, University of Oslo, P.O. Box 1029 Blindern, NO-0315 Oslo, Norway \\ ${ }^{4}$ Institute of Theoretical Astrophysics, University of Oslo, P.O. Box 1029 Blindern, NO-0315 Oslo, Norway \\ ${ }^{5}$ Instituto de Astrofísica de Canarias, 38205, C/Via Láctea s/n, La Laguna, Tenerife, Spain \\ ${ }^{6}$ Departimento de Astrofisica, Universidad de La Laguna, E-38205 La Laguna,Tenerife, Spain \\ ${ }^{7}$ INAF-Osservatorio Astrofisico di Catania, Via S. Sofia 78, I-95123 Catania, Italy \\ Received 2018 October 22; revised 2018 December 5; accepted 2018 December 5; published 2019 March 12
}

\begin{abstract}
We studied the physical parameters of the penumbra in a large and fully developed sunspot, one of the largest over the last two solar cycles, by using full-Stokes measurements taken at the photospheric Fe I $617.3 \mathrm{~nm}$ and chromospheric Ca II $854.2 \mathrm{~nm}$ lines with the Interferometric Bidimensional Spectrometer. Inverting measurements with the Non-LTE inversion COde (NICOLE) code, we obtained the three-dimensional structure of the magnetic field in the penumbra from the bottom of the photosphere up to the middle chromosphere. We analyzed the azimuthal and vertical gradient of the magnetic field strength and inclination. Our results provide new insights on the properties of the penumbral magnetic fields in the chromosphere at atmospheric heights unexplored in previous studies. We found signatures of the small-scale spine and intraspine structure of both the magnetic field strength and inclination at all investigated atmospheric heights. In particular, we report typical peak-to-peak variations of the field strength and inclination of $\approx 300 \mathrm{G}$ and $\approx 20^{\circ}$, respectively, in the photosphere, and of $\approx 200 \mathrm{G}$ and $\approx 10^{\circ}$ in the chromosphere. In addition, we estimated the vertical gradient of the magnetic field strength in the studied penumbra: we find a value of $\approx 0.3 \mathrm{G} \mathrm{km}^{-1}$ between the photosphere and the middle chromosphere. Interestingly, the photospheric magnetic field gradient changes sign from negative in the inner to positive in the outer penumbra.
\end{abstract}

Key words: Sun: chromosphere - Sun: magnetic fields - Sun: photosphere - sunspots

\section{Introduction}

Sunspots, which are the most prominent feature of the solar photosphere, are primary manifestations of the Sun's magnetism (Rempel \& Schlichenmaier 2011; van Driel-Gesztelyi \& Green 2015).

Penumbrae are integral parts of sunspots (Solanki 2003; Borrero \& Ichimoto 2011; Tiwari \& Hinode Review Team 2017). Earlier telescopic observations showed their complex filamentary nature, and penumbrae have since been the focus of numerous investigations. In recent years, observations taken with state-of-the-art space- and ground-based telescopes have been used to study the formation of penumbrae, for example, by Schlichenmaier et al. (2010, 2012), Romano et al. (2013, 2014), and Murabito et al. (2016, 2017, 2018), and to investigate their three-dimensional (3D) magnetic structure, for example, by Tiwari et al. (2013), Joshi et al. (2016, 2017a), and Tiwari \& Hinode Review Team (2017).

In the photosphere, penumbrae consist of nearly radially aligned filaments where strong and weak magnetic fields are interlaced with each other along the azimuthal direction. Indifferently from their position, penumbral filaments exhibit bright heads nearest to the umbral region and dark cores along their central axes (Tiwari et al. 2013). The filaments' heads show fields with the same polarity as the umbrae, which are enhanced $(\approx 1.5-2 \mathrm{kG})$ and more vertical $\left(\approx 35^{\circ}\right)$ than the fields observed along the axes of filaments. The latter fields are comparatively weaker $(\approx 1 \mathrm{kG})$ and more horizontal $\left(\approx 70^{\circ}\right)$ also with respect to the fields in filaments' tails, which are rather strong $(\approx 2-3.5 \mathrm{kG})$, and vertical fields of opposite polarity than umbrae (Tiwari et al. 2013). This field arrangement, which is usually described in terms of interlaced spines (more vertical and stronger fields) and intraspines (more horizontal and weaker field), is typical of all of the photospheric heights, but more prominent in the lower layers. The same field arrangement supports some models presented in the literature, such as the "uncombed" and "interlocking" penumbra models proposed by Solanki \& Montavon (1993) and Thomas et al. (2002), respectively. According to these models, the magnetic field strength in penumbrae decreases with atmospheric height, as well as its inclination (i.e., the field becomes more vertical when moving to higher atmospheric heights).

Recently, Balthasar (2018) presented an extensive review about the unsolved problems of the estimation of the magnetic field gradient in sunspots, exploring the different techniques used and the reasons for the discrepancy of the results presented in the literature. For example, Rueedi et al. (1995) found that the vertical gradient of the magnetic field decreases outward in the sunspot, with values of $0.1-0.3 \mathrm{G} \mathrm{km}^{-1}$ in the outer penumbra, and with the height in the atmosphere. Other authors, specifically Westendorp Plaza et al. (2001), Borrero \& Ichimoto (2011), and Orozco Suarez et al. (2015), reported signatures of magnetic fields forming canopylike structures in the middle and outer penumbrae, whereas Mathew et al. (2003), Sánchez Cuberes et al. (2005), and Balthasar \& Gömöry (2008) reported that the field simply increases with depth everywhere in sunspots. Tiwari et al. $(2013,2015)$ did not find any evidence for the canopylike structure but reported a reversed magnetic field gradient in the inner penumbra. Joshi et al. (2016) found that the magnetic field of the penumbra is more vertical in the upper chromosphere compared to that in 
the photosphere. Moreover, they found that the inclination of the field varies along the azimuthal direction in both the photosphere and upper chromosphere, whereas they reported small-scale spine/intraspine fluctuations of the field strength at photospheric heights only. Furthermore, Joshi et al. (2017a) found that the vertical gradient of the field strength displays large spatial fluctuations in the photosphere, changing its sign even on small scales. In particular, Joshi et al. (2017a) found that the vertical gradient of the field is always positive in spines, but in the part of the filament closer to the umbra, it is positive and surrounded by negative gradient at the sides of the filament in the lower atmospheric layers, being always negative in the upper layers. Joshi et al. (2017a) also reported that the tail of filaments has a positive field gradient both in the lower and upper photospheric layers.

It is worth nothing that the above current understanding of the small-scale 3D magnetic structure of penumbral filaments derives from analysis of data taken through several photospheric Fe I, SiI, and CaI lines, ${ }^{8}$ but one chromospheric diagnostic only, the He I triplet at $1083.0 \mathrm{~nm}$. The latter spectral region, which offers a unique tool to study photospheric and chromospheric fields simultaneously, is strongly influenced by EUV coronal irradiation penetrating the atmosphere deep into the upper chromosphere, to a height that hydrostatic atmosphere models indicate to be around $2000 \mathrm{~km}$ above the photosphere (Avrett et al. 1994), where the He I $1083.0 \mathrm{~nm}$ spectrum is thought to be formed. From analysis of penumbral observations taken in the spectral region including the $\mathrm{He}$ I triplet, Joshi et al. (2017b) reported the presence of the smallscale spine/intraspine structure of the magnetic field strength and inclination at photospheric heights between $\log \tau=0$ and $\log \tau=-2.3$, where $\log \tau$ is the $\log$ arithm to the power of 10 of the optical depth and of the same but attenuated structure only for the field inclination in the chromosphere sampled with the He I triplet. However, because of the current limited observations and modeling capabilities for investigating the chromospheric heights, the magnetic field structure of penumbrae above the photosphere remains poorly understood.

Recently, Joshi \& de la Cruz Rodríguez (2018) studied the variations of magnetic field in a sunspot from the photosphere to the chromosphere by using a different chromospheric diagnostic than in the previous works, specifically observations taken in the Ca II $854.2 \mathrm{~nm}$ line. According to Quintero Noda et al. (2016), the Ca II $854.2 \mathrm{~nm}$ line is mostly sensitive to the atmospheric layers enclosed in the range $\log \tau=[0,-5.5]$, which is the atmosphere from the bottom of the photosphere to the middle chromosphere, up to heights that hydrostatic models estimate to be of about $1000-1500 \mathrm{~km}$ above the photosphere. In addition, Quintero Noda et al. (2017) found that the sensitivity of the $\mathrm{Ca}$ II line to the atmospheric parameters, in particular the photospheric sensitivity to the vector magnetic field, largely increases when additional photospheric spectral lines are analyzed.

In their study, Joshi \& de la Cruz Rodríguez (2018) analyzed the variation of the atmospheric parameters associated with oscillations manifested as umbral flashes and penumbral waves, but they also estimated the average properties of the vertical gradient of the penumbral magnetic field between the photosphere and chromosphere. They did not describe the fine-scale

\footnotetext{
8 Specifically, the Fe I 630.1, Fe I 630.2 nm, Fe I 1564.8 nm, Fe I 1565.3 nm, Fe I $1078.3 \mathrm{~nm}$, Si I $1078.4 \mathrm{~nm}$, Si I $1078.7 \mathrm{~nm}$, Si I $1082.7 \mathrm{~nm}$, and Ca I $1083.3 \mathrm{~nm}$ lines.
}

structure of the observed penumbral field and just reported on a decrease of the magnetic field with a rate of $-0.5 \mathrm{G} \mathrm{km}^{-1}$ in the vertical direction, in agreement with values in the literature, ranging from -0.3 to $-1.0 \mathrm{G} \mathrm{km}^{-1}$. Earlier studies by SocasNavarro (2005a, 2005b) of photospheric and chromospheric observations taken at the $850 \mathrm{~nm}$ spectral range, including Ca II data as well, only showed the complex topology of sunspot magnetic field with areas of opposite-sign torsion and twist, suggestive of flux ropes of opposite helicity coexisting together in the same spot, with no further details on the fine-scale structure of the field because of the spatial resolution of the analyzed data.

In this article, we study the 3D magnetic structure of the penumbra in a large and fully developed sunspot, as derived from simultaneous inversion with the NICOLE code of photospheric Fe I $617.3 \mathrm{~nm}$ and chromospheric Ca II $854.2 \mathrm{~nm}$ data. Although the latter data provide an excellent tool for investigating the chromospheric environment (Cauzzi et al. 2008), to date the Ca II data have been less explored than other diagnostics to study penumbral regions. In this light, the results presented here offer new information on the properties of the chromospheric magnetic field in penumbral regions, especially concerning the geometrical heights of different parts of penumbral filaments and the penumbral structure at chromospheric heights above $\log \tau=-2.3$, which were not analyzed in previous studies (Tiwari \& Hinode Review Team 2017).

\section{Data and Methods}

\subsection{Observations}

We analyzed data acquired on 2016 May 20, with the Interferometric Bidimensional Spectrometer (IBIS; Cavallini 2006) at the Dunn Solar Telescope of the National Solar Observatory. These observations concern the large and mature sunspot of active region NOAA 12546 at the time located near disk center at (S07, W07) and $\mu \approx 0.97$, where $\mu$ is the cosine of the heliocentric angle. The data consist of full-Stokes measurements taken from 13:53 UT to 18:17 UT along the Fe I $617.3 \mathrm{~nm}$ and $\mathrm{Ca}$ II $854.2 \mathrm{~nm}$ lines, with a cadence of $48 \mathrm{~s}$. The spectral ranges of the Fe I and Ca II measurements span from $617.1 \mathrm{~nm}$ to $617.5 \mathrm{~nm}$ and from $853.6 \mathrm{~nm}$ to $854.8 \mathrm{~nm}$, respectively. The polarimetric data were taken at 21 spectral points in each line, with a spectral sampling of $20 \mathrm{m \AA}$ and $60 \mathrm{m \AA}$ for the Fe I $617.3 \mathrm{~nm}$ and $\mathrm{CaII} 854.2 \mathrm{~nm}$ lines, respectively. The field of view $(\mathrm{FOV})$ was $500 \times 1000$ pixels with a pixel scale of 0 ." 08 . The above measurements were complemented with simultaneous broadband images taken at $633.32 \pm 5 \mathrm{~nm}$ on the same FOV and with the same exposure time of the polarimetric data. For further details on the analyzed observations, see Stangalini et al. (2018).

The sunspot was observed with the adaptive optic system (AO; Rimmele 2004) of the Dunn Solar Telescope locked and running on the center of the umbral region. The spatial resolution of the data is 0 !" 16 and 0 "! 23 for the Fe I and Ca II line measurements, respectively.

In this article, we show results derived from analysis of the best scan in the long-duration data set obtained during the observations (318 scans available). According to rms values in quiet Sun regions and all the measurements taken along the sampled lines, the best scan corresponds to the nr 191 of the series, whose acquisition started at 16:52 UT. 

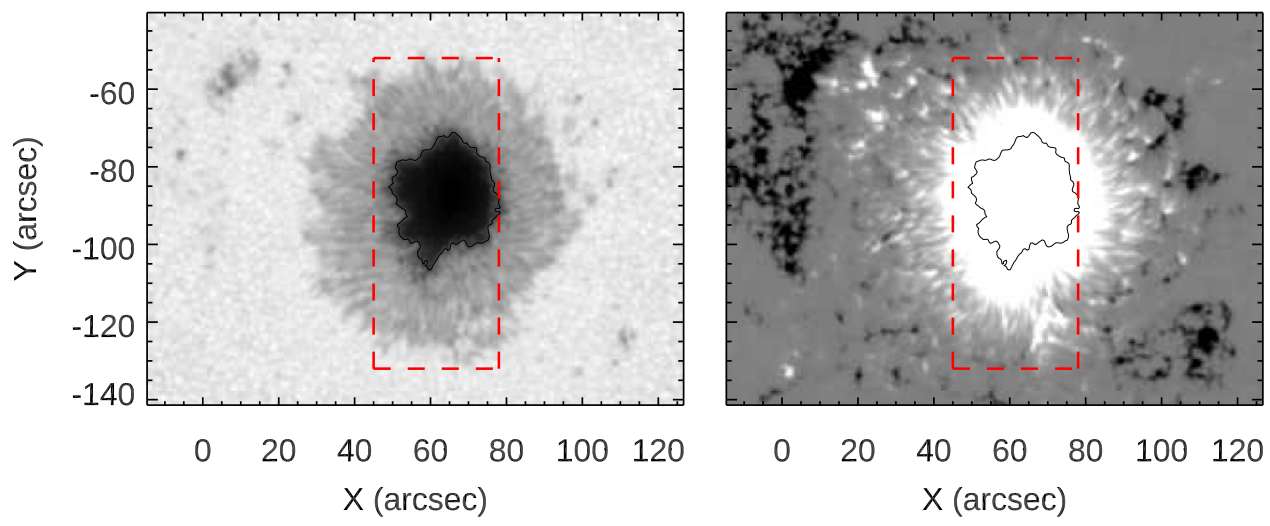

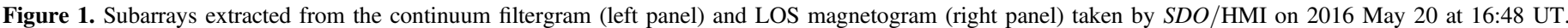

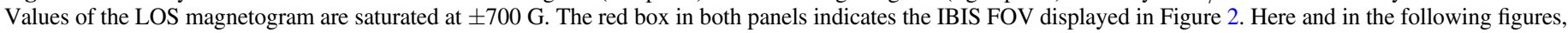
solar north is at the top, and west is to the right.

The observations were calibrated by using standard procedures for flat-fielding, dark subtraction, and polarimetric calibration. Moreover, the data were restored for seeinginduced degradation left over by the telescope adaptive optic system with application of the Multi-Object Multi-Frame Blind Deconvolution (MOMFBD; van Noort et al. 2005) technique to the calibrated measurements. In addition to the seeing degradation, the observations analyzed in our study also suffer from stray-light contamination due to instrument design. According to Reardon \& Cavallini (2008), at the Fe I and Ca II spectral ranges, the level of the IBIS stray light is $\approx 2 \%$ and $1.3 \%$ of the instrumental transmittance, respectively. We account for this degradation of the data in the NICOLE inversion, which is described later, by allowing the macroturbulence parameter to vary in the processing, without considering the instrumental stray-light point-spread function in the computations.

Active region NOAA 12546 appeared on the east solar limb on 2016 May 13. It consisted of an isolated, large, and fully developed sunspot with a leading unipolar magnetic field configuration ( $\alpha$-type) of positive polarity and a nearby plage region with trailing negative polarity fields. It is worth nothing that this is a peculiar sunspot because of its dimension and magnetic field strength (exceeding $4 \mathrm{kG}$, as resulting from Hinode/the spectopolarimeter of the Solar Optical Telescope (SOT-SP; Tsuneta et al. 2008; Lites et al. 2013) level 2 data, not shown here). In fact, a recent work by Livingston \& Watson (2015) showed that, in the 2010-2015 period, there were only a few sunspots with an umbral magnetic field exceeding $3500 \mathrm{G}$.

Figure 1 shows subarrays extracted from the continuum filtergram and line-of-sight (LOS) magnetogram taken by the Helioseismic and Magnetic Imager (HMI; Scherrer et al. 2012) onboard the Solar Dynamics Observatory (SDO; Pesnell et al. 2012) on 2016 May 20, at 16:48 UT (i.e., close in time to the IBIS observations analyzed in our study). The SDO/HMI observations were taken in the $\mathrm{Fe}$ I $617.3 \mathrm{~nm}$ line with a pixel scale of about $0 . " 5$.

The red box in Figure 1 displays the FOV of the IBIS observations, whose examples are given in Figure 2. The IBIS FOV, which is centered on the sunspot barycenter, covers the entire umbra and most of the penumbral region of the studied spot, especially in the north and south directions.

Figure 2 (top panels) shows IBIS intensity images obtained in the continuum and in the core of the $\mathrm{Fe}$ I $617.3 \mathrm{~nm}$ and $\mathrm{Ca}$ II
$854.2 \mathrm{~nm}$ lines. To make the small-scale structures in the FOV clearer, we display both the Fe I and Ca II line-core intensity maps with the values normalized to the local continuum intensity.

The IBIS Fe I line-continuum intensity data (Figure 2(a)), and more thoroughly the cotemporal SDO/HMI continuum filtergram in Figure 1, show that the studied penumbra is almost circular and rather homogeneous, except for a small sector in the south-west direction (shown by the black arrow in panel a). The Fe I line-core intensity data (Figure 2(b)) display different average values in the inner and outer parts of the penumbra, with a decrease of the line-core intensity values when moving away from the spot center. The Ca II linecontinuum intensity map (panel c) shows minute deviations to the corresponding Fe I map. The intensity map obtained in the line-core of the Ca II data (Figure 2(d)) shows the superpenumbra (Loughhead 1968) near the edges of the FOV, especially at the north-west and south-east directions. Unfortunately, the IBIS FOV covers only a small part of the superpenumbral region. The same data also display two bright regions, located to the north-east and south-west sides of the FOV (shown by the two blue arrows in Figure 2(d)), the latter corresponding with a region where the distribution of interlaced filaments is less regular than in other penumbral sectors (marked by the black arrow in Figure 2(a)).

Figure 2 (bottom panels) shows the maps of the mean circular $C_{p}$ and linear $L_{p}$ polarization signals derived from the $\mathrm{Fe}$ I and Ca II line data. These maps were computed pixelwise following Guglielmino et al. (2012) by using the formulae:

$$
\begin{gathered}
C_{p}=\frac{1}{10\left\langle I_{c}\right\rangle} \sum_{i=5}^{15} \epsilon_{i}\left|V_{i}\right| \\
L_{p}=\frac{1}{10\left\langle I_{c}\right\rangle} \sum_{i=5}^{15} \sqrt{Q_{i}^{2}+U_{i}^{2}}
\end{gathered}
$$

where $\left\langle I_{c}\right\rangle$ is the average value of Stokes $I$ in the line-continuum in a quiet region, $\epsilon=1$ for the first five spectral positions of the line sampling, $\epsilon=-1$ for the last five positions, and $\epsilon=0$ for the line center position, and $i$ runs from the 5 th to the 15th wavelength positions.

The $C_{p}$ (Figure 2(e)) and $L_{p}$ (Figure 2(f)) maps derived from the Fe I data clearly show the spine/intraspine structure of the penumbra observed in the photosphere. However, signatures of 

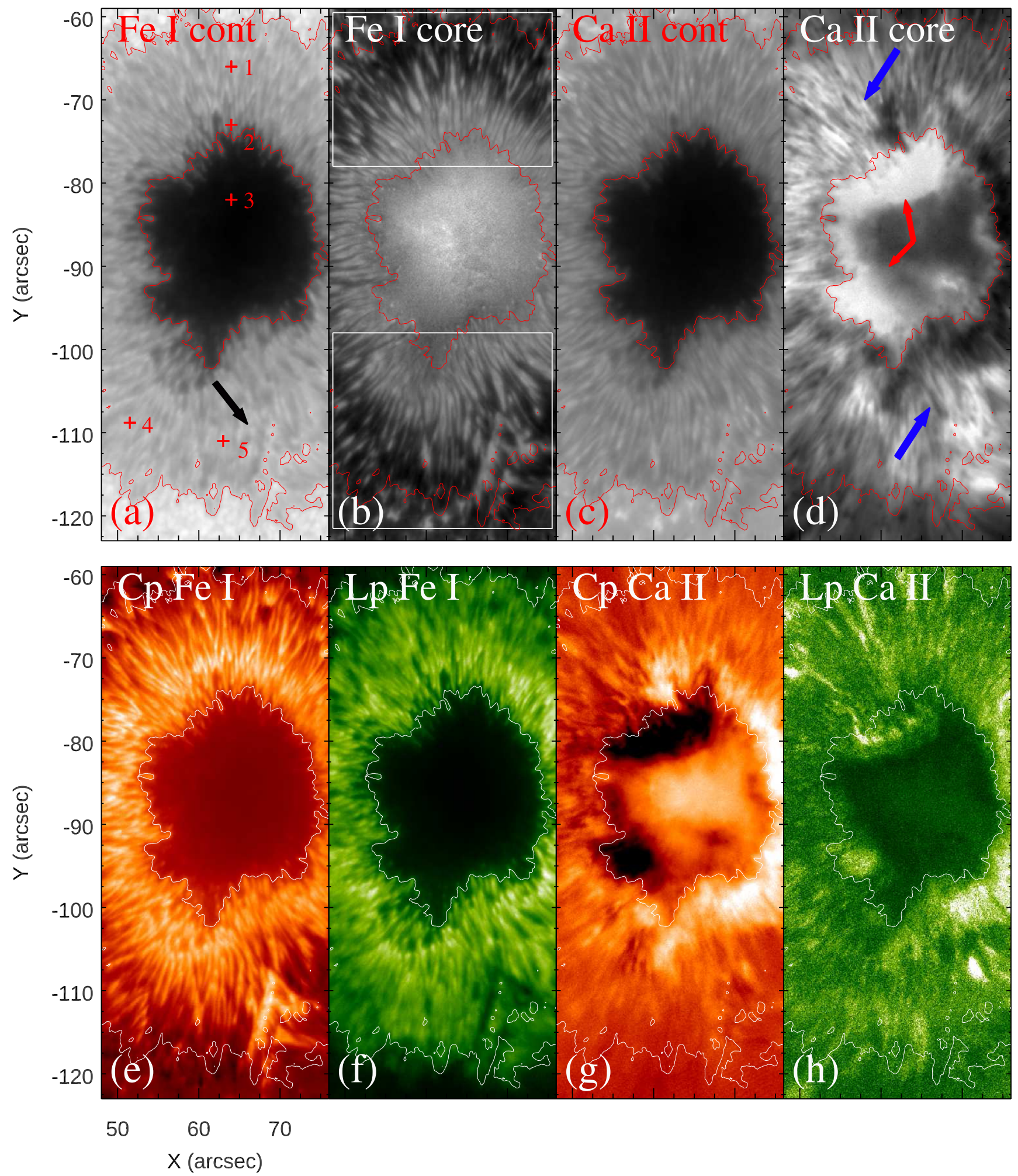

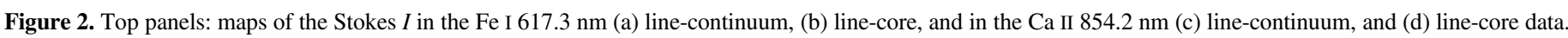

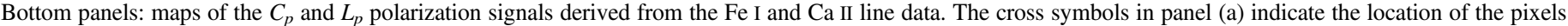

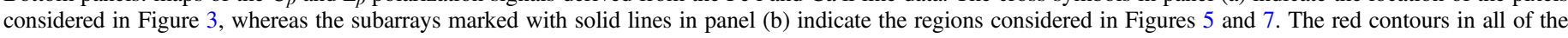

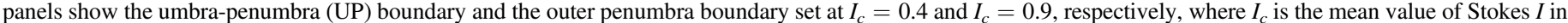
a quiet Sun region. The arrows indicate the regions described in the text.

the same structure are also found in both the $C_{p}$ (Figure 2(g)) and $L_{p}$ (Figure 2(h)) maps obtained from the Ca II data. The intense brightening in the $C_{p}$ map localized near the UP boundary (see the two red arrows in panel Figure 2(d)), defined by the threshold in the Fe I line-continuum intensity $I_{c}=0.4$, is due to wave processes that will be analyzed in a future article. 


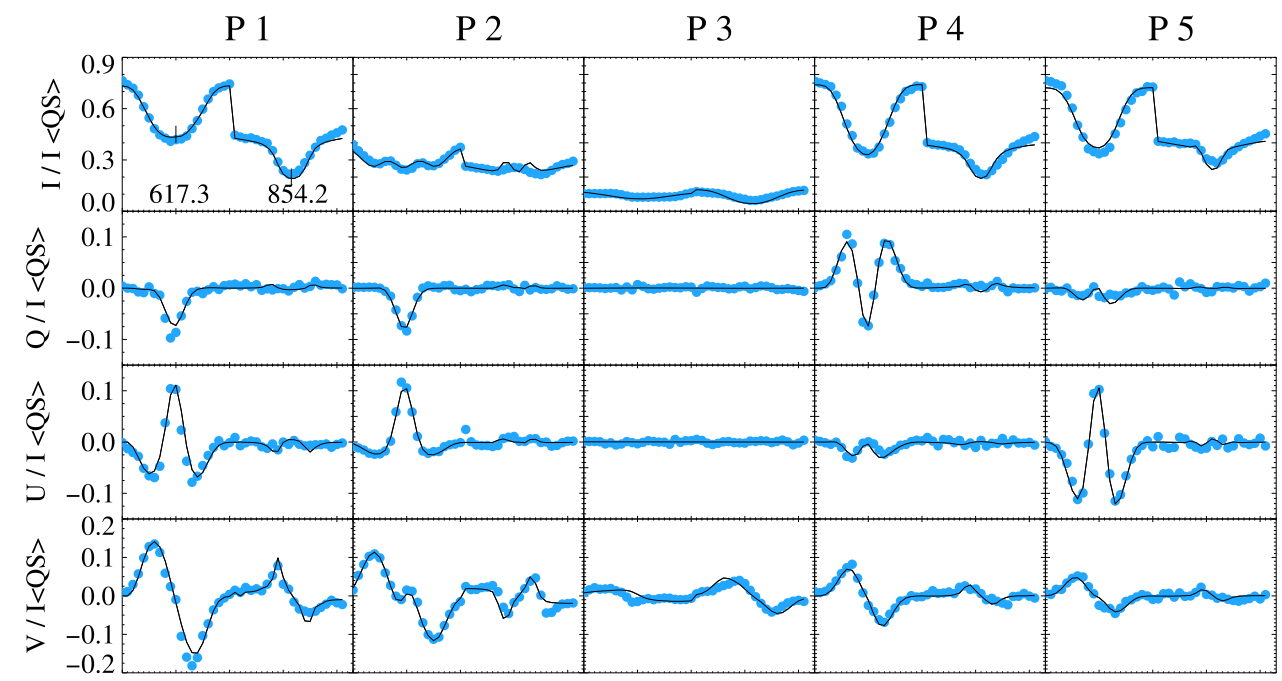

Figure 3. Examples of observed (filled light blue circles) and inverted (black solid line) Stokes profiles for the image pixels belonging to the penumbra (P1, P4, and P5), UP boundary (P2), and umbra (P3) at the locations shown in Figure 2(a).

\subsection{Data Inversion}

We used NICOLE (Socas-Navarro et al. 2015) to derive the physical parameters in the observed region at the atmospheric heights sampled by the analyzed data. NICOLE solves the nonLTE radiative transfer in a plane-parallel geometry under statistical equilibrium, by assuming complete redistribution in angle and frequencies of scattered photons to compute intensities. We refer the reader to the above article for further details.

We show in the following section the results derived from inversions of the Fe I and Ca II lines simultaneously. We used five equidistant nodes located from $\log \tau=-7$ and $\log \tau=1$ for temperature, three nodes for each component of the vector magnetic field $\left(B_{x}, B_{y}\right.$, and $\left.B_{z}\right)$, two nodes for the LOS velocity, and one node for both the microturbulence and macroturbulence. This setting of the nodes, which resulted from test inversions performed to best reproduce the observed spectra, is compliant with that used by Robustini et al. (2018) to derive the magnetic field topology in chromospheric regions hosting jets. We performed the inversions assuming as the initial guess model an atmosphere model C (FALC) by Fontenla et al. (1993) modified with a constant value of $1.5 \mathrm{kG}$ for $B_{z}$.

Figure 3 shows examples of the observed and inverted Stokes profiles for five pixels located at different positions in the FOV, specifically in the umbra, in the UP boundary, and at three locations in the penumbra. The position of these pixels is marked with crosses in Figure 2(a). For all of the considered penumbral pixels, the inverted profiles match well the observed ones, especially when considering the $\mathrm{Fe}$ I data that have Stokes $Q, U$, and $V$ signals with maximum amplitude of about $\approx 10 \%, 10 \%$, and $15 \%$ of the Stokes $I$ values in quiet Sun regions, respectively. The Stokes profiles measured in the Ca II line are characterized by lower Stokes $Q$ and $U$ signals than reported above for the Fe I data, but the values measured at the Ca II are still above the noise level $\left(\approx 5 \times 10^{-3}\right)$ of the data (typical noise level is $10^{-3}$; see, e.g., Lagg et al. 2017 and Stangalini et al. 2018). Therefore, the inverted profiles depict reasonably well the observed profiles for all of the analyzed penumbral pixels. On the contrary, for some pixels in the UP boundary and in the umbra, such as those considered in Figures 2 and 3, the measured Stokes profiles are extremely reduced and even distorted with respect to those reported above. For example, the Stokes profiles of the UP boundary pixel considered in Figure 3 show emission line reversals in the blue and red lobes of both the $\mathrm{Fe}$ I and $\mathrm{Ca}$ II lines. These distorted profiles are likely due to either solar transient events and waves affecting the measurements, or seeing-induced aberrations that are not accounted for during the observations and with the data processing. At the UP boundary and in the umbra, the Stokes $Q, U$, and $V$ signals are very low. Although the Stokes $Q$ and $U$ signals of the chromospheric data in UP pixels are still above the data noise, for some pixels in the umbra, the same signals are close to the noise level, impeding any accurate data inversion. On the basis of these properties of the analyzed data, we focused our study on the penumbral region and ignored any results derived from the data inversion that concern the transverse and vertical components of the umbral magnetic field. We also considered results for the transverse component of the field derived from the data inversion of the UP region with caution. It is worth noting that our data selection is supported by the recent results by Felipe et al. (2018). Those authors studied the accuracy of the atmospheric parameters derived from inversion of spectropolarimetric data, including $\mathrm{Ca}$ II observations obtained with the scanning of spectral lines, such as the one analyzed in our study. By using synthetic data from magnetohydrodynamic simulations, Felipe et al. (2018) showed that inversion of such data provides an unreliable characterization of the thermodynamic properties of the atmosphere, when the measured Stokes profiles have apparent wavelength shifts and spurious deformations due to propagation of waves in sunspots, as for some of the data analyzed in our study.

Because the studied sunspot was observed close to disk center, the vector magnetic field was considered as returned from the data inversion - that is, the field values derived from the inversion were not transformed into the solar frame of reference. The $180^{\circ}$ ambiguity in the azimuth direction was also not resolved.

\subsection{Response Functions}

To assess the range of atmospheric heights in which the analyzed data are sensitive to perturbations of the magnetic 

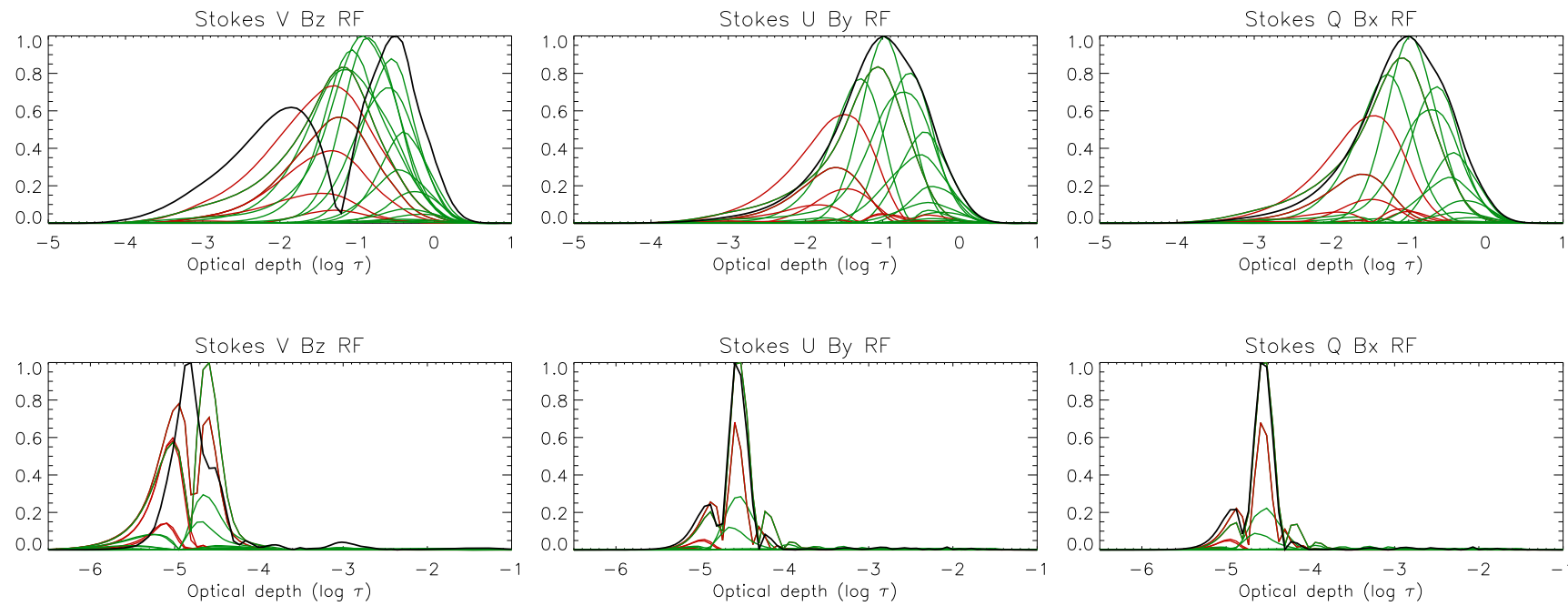

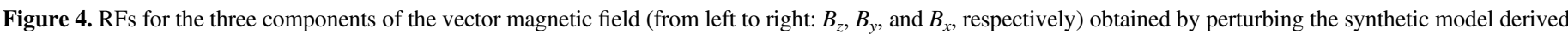

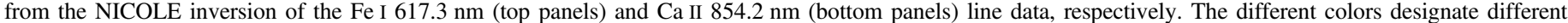

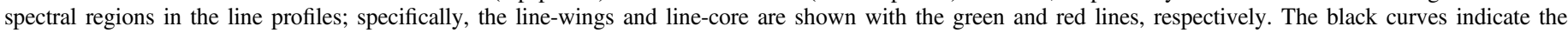
wavelength-integrated RFs. Each RF is normalized to the maximum value of the RF obtained for the given Stokes parameter considered.

field, we computed the response functions (RFs) for the Stokes profiles to perturbations of the atmosphere model derived from the data inversion. We followed the method of Quintero Noda et al. (2016). In particular, we computed $\mathrm{RF}_{B_{i}}^{S}$, where $\mathrm{S}$ represents Stokes $Q, U$, and $V$, and $B_{i}$ indicates one of the three magnetic field components, $B_{x}, B_{y}$, and $B_{z}$. We computed the RFs for a penumbral area of $10 \times 10$ pixels by averaging the Stokes profiles therein and normalizing the obtained results with respect to the maximum value of the RF obtained for each profile. For a given Stokes parameter, optical depth, and wavelength, RF values close to unity indicate that the measurements of the corresponding Stokes-parameters are quite responsive to perturbations of the line-forming atmosphere, whereas low or null values of RFs indicate that the Stokes measurements are unaffected by inhomogeneities in the atmospheric parameters, because of changes of the magnetic field. This implies that the data inversion cannot provide reliable information about atmospheric physical quantities in the line-forming regions characterized by low RFs values, because these regions lie outside the sensitivity range of the analyzed data to perturbation of atmospheric parameters.

Figure 4 shows plots for some of the estimated RFs that are representative of all the RFs computed on the atmosphere model returned by the NICOLE inversion. In particular, top panels display the RFs for the three components of the magnetic field represented by the Stokes $Q, U$, and $V$ profiles of the synthetic Fe I data derived from the inverted atmosphere. These panels show that the analyzed line is sensitive to perturbations of the magnetic field components at atmospheric heights ranging from $\log \tau=0.5$ to $\log \tau=-4$. However, the regions around $\log \tau=0$ and those above $\log \tau=-2$ show rather low RF values, suggesting that we cannot retrieve reliable information on the properties of the magnetic field at those atmospheric heights by the analyzed Fe I data. Bottom panels in Figure 4 display the RFs derived from analysis of the synthetic Ca II data. These panels show that the latter data are sensitive to variations of the magnetic fields at different atmospheric heights, ranging from $\log \tau=-1$ to $\log \tau=-6$. High RF values are obtained for heights between $\log \tau=-4$ to $\log \tau=-6$, and the maximum of sensitivity is at $\log \tau \approx$ -4.6 .

According to these results, we show in the following section estimates of the magnetic field vector at four atmospheric heights corresponding to local maxima of the estimated RFs in the photosphere at $\log \tau=-0.5,-1.0$, and -1.5 , and in the chromosphere at $\log \tau \approx-4.6$.

\section{Results}

Figure 5 displays subarrays extracted from the maps of the magnetic field strength and inclination returned from the data inversion. These subarrays refer to the regions marked with solid lines in Figure 2(b), which are labeled A and B in the following paragraphs on the basis of their position north and south with respect to the barycenter of the spot.

The maps of the magnetic field strength and inclination at $\log \tau=-0.5,-1.0$, and -1.5 show the well-known spine/ intraspine structure observed in photospheric penumbrae; locations where these small-scale features are seen more easily are enclosed by black ellipses overplotted to the inclination map at $\log \tau=-1$. Both the studied quantities display rather similar values at all the considered photospheric heights. The maps of the field strength and inclination referring to the chromospheric height $\log \tau=-4.6$ show a pattern reminiscent of the small-scale structure observed in the photosphere, but with rather different values than those estimated therein. Table 1 summarizes the mean and standard deviation of the values of the magnetic field strength and inclination measured inside and outside the penumbra, by pointing out the average variation of these quantities when moving from the photosphere to the chromosphere. For these estimates, the penumbra was identified as the region where Stokes $I$ of the linecontinuum is between 0.4 and 0.9 the average value in quiet Sun regions. It is worth noting that the values of the photospheric magnetic field strength listed in Table 1 are comparable to those average magnetic field strength values usually reported for the umbra of sunspots (Livingston \& Watson 2015). We stress here that the results presented in the following paragraphs should be read in light of this. 


$$
\log \tau=-0.5 \quad \log \tau=-1 \quad \log \tau=-1.5 \quad \log \tau=-4.6
$$
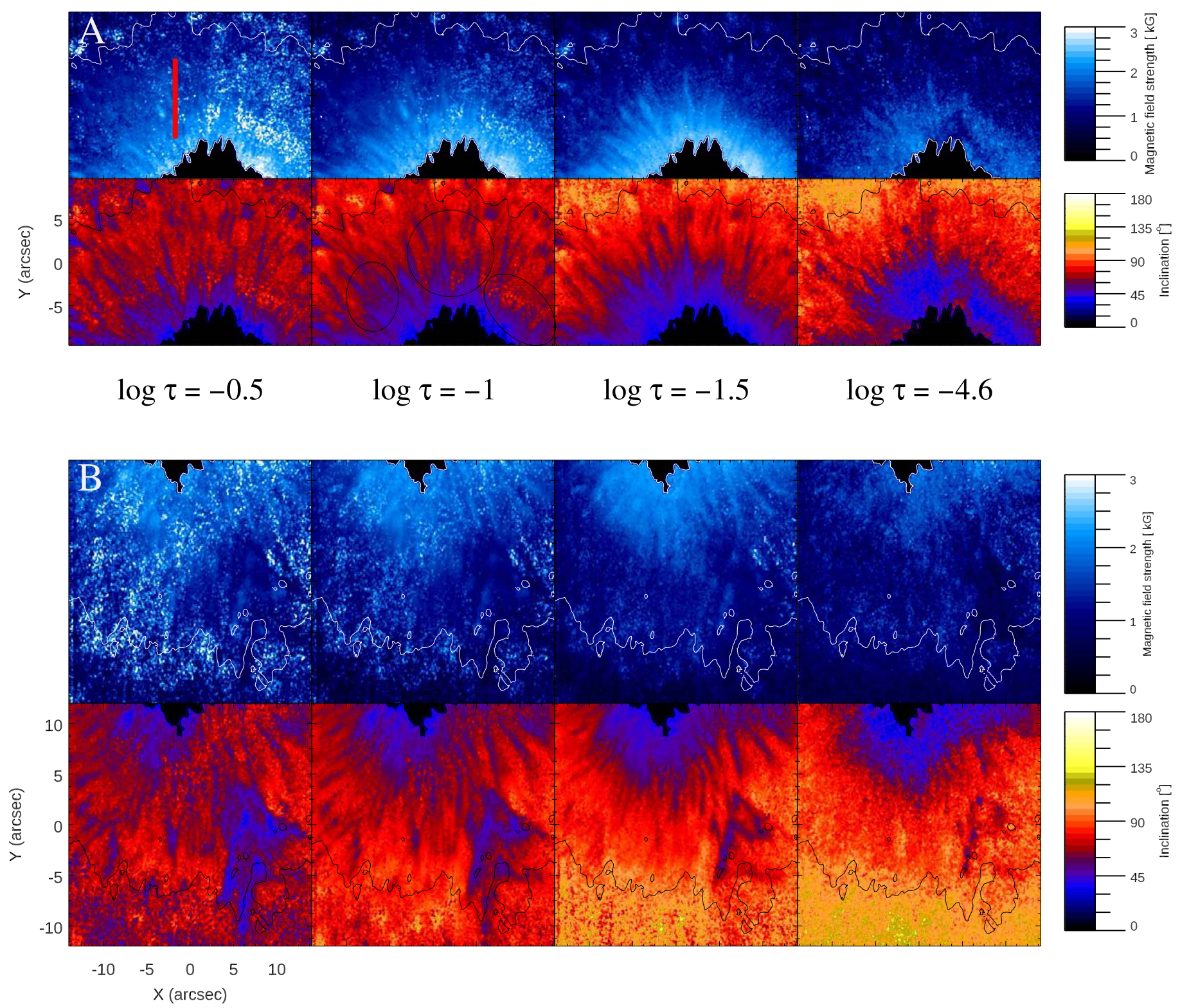

Figure 5. Maps of the magnetic field strength (first and third rows) and field inclination (second and fourth rows) derived from NICOLE inversion of the Fe I 617.3 nm and Ca II $854.2 \mathrm{~nm}$ line data. The results shown here refer to the two subarrays displayed in Figure 2(b), located in the northern and southern sectors of the IBIS FOV. Black and white contours represent the edge of the umbra and the outer penumbra in the photosphere, as defined in Figure 2. The region marked red in the top-left panel is used for the analysis reported in Figure 6. From left to right: the four maps in each row correspond to the results derived from the data inversion at $\log \tau=-0.5,-1 .,-1.5$, and -4.6 . The black ellipses enclose locations where the small-scale structures described in the main text are seen more clearly.

The maps of the inclination at the photospheric heights $\log \tau=-0.5$ and -1 display a radially homogeneous distribution of the field, with interlaced channellike features hosting more vertical and more horizontal fields, respectively. The regular distribution of the inclination fails in the southwest sector of the penumbra shown in subarray B of Figure 5, where the filaments are rather inhomogeneous. The maps referring to the chromospheric height at $\log \tau=-4.6$ display fields with higher strength in the inner part of the penumbra compared to the fields in the outer part, which, however, show slight variations along the azimuthal direction.

Figure 6 displays the variation of the magnetic field strength (left panel) and inclination (right panel) in the region marked red in the subarray A of Figure 5. This region is 4 pixels wide and 171 pixels long. We computed the variation of the field strength and inclination along the vertical region moving from the inner to the outer penumbra, by averaging the values of the magnetic quantities at same distance from the spot barycenter. The distance shown in the $x$-axis was computed with respect to the UP boundary, moving away from the umbra. Black, orange, blue, and magenta lines represent field values estimated at the atmospheric heights $\log \tau=-0.5,-1.0,-1.5$, and -4.6 , respectively.

When moving from the inner to the outer part of the penumbra, the field strength (left panel of Figure 6) decreases in the photosphere from 2.3 to $1.5 \mathrm{kG}$ at $\log \tau=-0.5$, and 

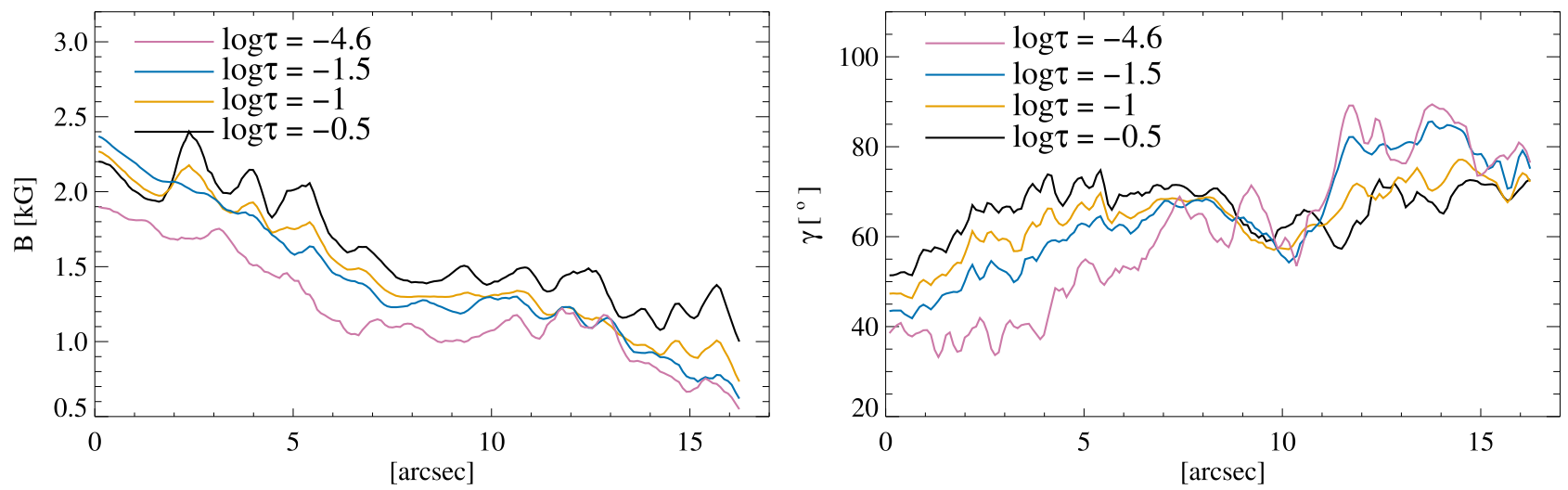

Figure 6. Variation of the magnetic field strength (left panel) and inclination (right panel) in the vertical region marked red in Figure 5. Each profile is obtained by averaging the field values at corresponding distance from the spot barycenter. The distance shown in the $x$-axis is computed with respect to the UP boundary, moving away from the umbra. Black, orange, blue, and magenta represent the field values estimated at $\log \tau=-0.5,-1.0,-1.5$, and -4.6 , respectively.

Table 1

Magnetic Field Strength and Inclination

\begin{tabular}{lcccc}
\hline \hline $\log \tau$ & $\begin{array}{c}\bar{B}_{p} \pm \sigma \\
(\mathrm{kG})\end{array}$ & $\begin{array}{c}\bar{B}^{\text {out }} \pm \sigma \\
(\mathrm{kG})\end{array}$ & $\begin{array}{c}\bar{\gamma}_{p} \pm \sigma \\
\left({ }^{\circ}\right)\end{array}$ & $\begin{array}{c}\bar{\gamma}^{\text {out }} \pm \sigma \\
\left({ }^{\circ}\right)\end{array}$ \\
\hline-0.5 & $1.7 \pm 0.4$ & $1.6 \pm 0.4$ & $60 \pm 9$ & $63 \pm 9$ \\
-1 & $1.6 \pm 0.4$ & $0.9 \pm 0.4$ & $61 \pm 9$ & $78 \pm 9$ \\
-1.5 & $1.6 \pm 0.4$ & $0.8 \pm 0.4$ & $64 \pm 9$ & $95 \pm 9$ \\
-4.6 & $1.2 \pm 0.5$ & $0.8 \pm 0.5$ & $68 \pm 15$ & $104 \pm 15$ \\
\hline
\end{tabular}

Note. $\bar{B}_{p} \pm \sigma$ : Mean \pm standard deviation of the values of the magnetic field strength in the penumbra. $\bar{B}^{\text {out }} \pm \sigma$ : Mean \pm standard deviation of the values of the magnetic field strength outside the penumbra. $\bar{\gamma}_{p} \pm \sigma$ : Mean \pm standard deviation of the values of the magnetic field inclination in the penumbra. $\bar{\gamma}^{\text {out }} \pm \sigma$ : Mean \pm standard deviation of the values of the magnetic field inclination outside the penumbra.

from 1.3 to $0.7 \mathrm{kG}$ at $\log \tau=-1.5$; in the chromosphere, it decreases from $\approx 1.1$ to $0.6 \mathrm{kG}$ at $\log \tau=-4.6$. The inner part of the penumbra is characterized by magnetic fields that become more vertical with the atmospheric height. In particular, close to the UP boundary, the values of the field inclination (right panel of Figure 6) are around $50^{\circ}$ and $40^{\circ}$ at $\log \tau=-0.5$ and $\log \tau=-4.6$, respectively. In contrast, in the outer part of the penumbra, the field becomes more horizontal with the atmospheric height. The values of the field inclination measured in the photosphere and chromosphere differ of about $20^{\circ}$, reaching about $90^{\circ}$ in the chromosphere.

To further analyze the small-scale spine/intraspine structure of the penumbra in the photosphere and chromosphere, we considered the properties of the penumbral magnetic field at different distances from the UP boundary. In particular, we estimated the variation of the LOS component of the field and inclination along the three arcs shown in the Fe I linecontinuum intensity map in Figure 7 (left panel). One arc lies in the outer penumbra (hereafter A1), whereas the other two (hereafter A2 and A3) are located in the inner part of the penumbra. We consider here the LOS component of the field, instead of the magnetic field strength, because the former quantity shows the spine/intraspine structure more clearly. We estimated the variation of the field parameters with respect to the azimuthal distance along each arc. The filaments in the northern part of the penumbra sampled by A1 and A2 are uniformly distributed along azimuthal direction, whereas those in the southern part of the penumbra sampled by A3 are less uniformly arranged; see, for example, the region centered at $[x, y]=\left[45^{\prime \prime}, 115^{\prime \prime}\right]$.

Figure 7 (right panels) displays the LOS component of the magnetic field (top panels) and inclination (bottom panels) along the three studied arcs. The small-scale fluctuation of the field along A1 in the outer penumbra is weak but still detectable at all considered atmospheric heights. In the photosphere at $\log \tau=-1$ and $\log \tau=-1.5$, the $\operatorname{LOS}$ component of the field shows interlaced higher and lower values with peak-to-peak changes of $\approx 300 \mathrm{G}$; this field fluctuation is reduced to changes of $\approx 150-200 \mathrm{G}$ but is yet evident at $\log \tau=-4.6$. The values of the LOS component of the field along A 2 and A 3 in the inner penumbra exhibit similar trends as those obtained along A1 in the outer penumbra, but with stronger field values. The field variation along A3 displays a decrease of the average value of the field at the azimuthal distance $\approx 10^{\circ}$ in the south-west penumbral sector.

Figure 7 (bottom panels) shows that, along A2 and A3 in the inner penumbra, the magnetic field inclination decreases when passing from the photosphere to the chromosphere. The peakto-peak variation of the values of the field inclination in these regions decreases from $\approx 20^{\circ}$ at $\log \tau=-0.5$ to $\approx 10^{\circ}$ at $\log \tau=-4.6$. The more vertical chromospheric field depicted can be explained with the presence of magnetic canopy at different heights. In detail, when comparing photospheric and chromospheric estimates of the field inclination at the same disk position, we are considering field lines connected to different foot-points in the penumbra (Jafarzadeh et al. 2017). The photospheric pattern of the field inclination along A1 in the outer penumbra corresponds well to that observed for the inclination in the middle chromosphere. In addition, along A1 the magnetic field becomes more horizontal with the atmospheric height, by assuming values of $\approx 55^{\circ}-60^{\circ}$ in the photosphere and up to $90^{\circ}$ in the chromosphere.

We then studied the vertical gradient of the magnetic field strength, in order to discuss our results with respect to those in the literature. Following Joshi et al. (2017a), we defined the 

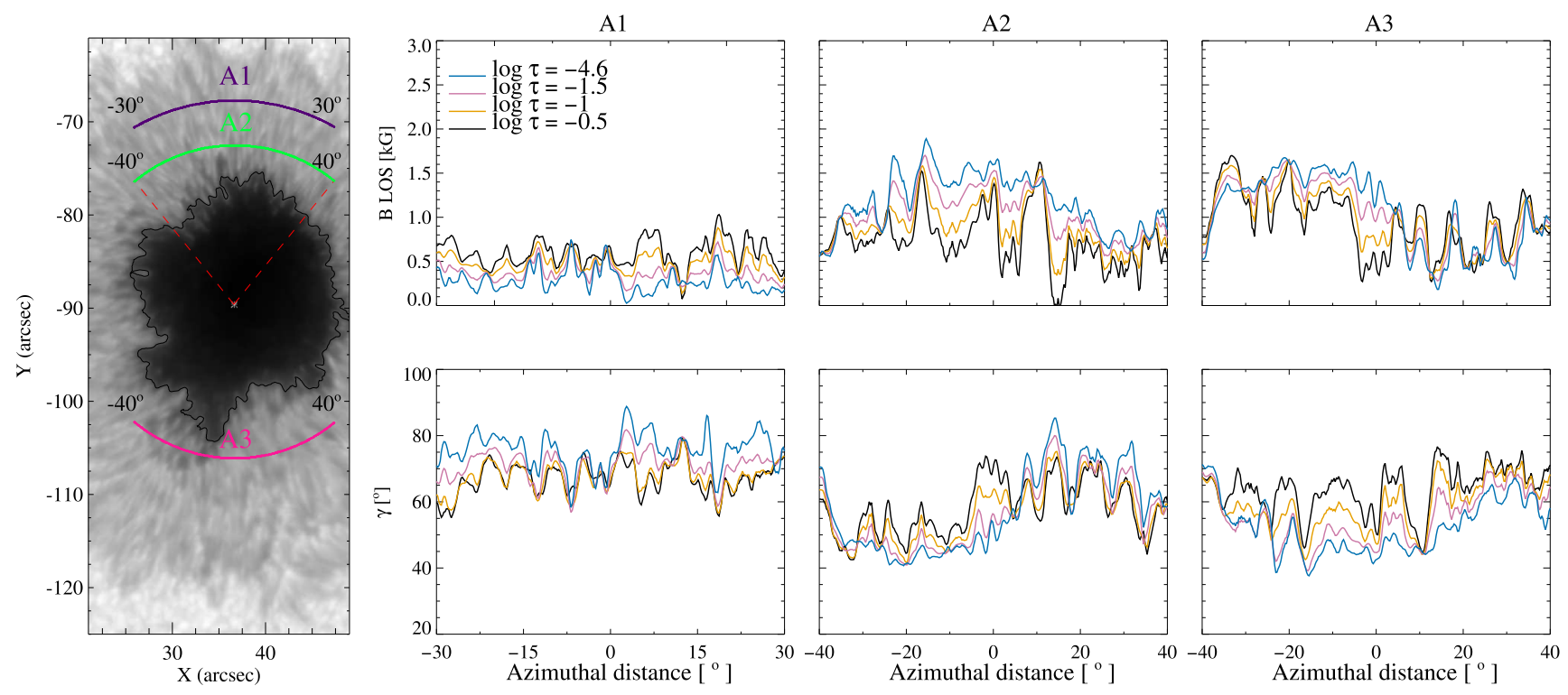

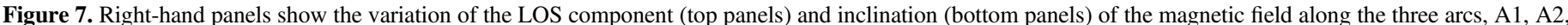

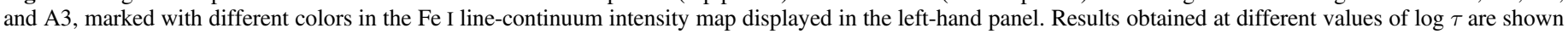
with the various colors as displayed in the legend. Find more details in Section 3.

vertical gradient of the magnetic field strength as:

$$
\left(\frac{\Delta B}{\Delta \log \tau}\right)_{a, b}=\frac{(\Delta B)_{a, b}}{(\Delta \log \tau)_{a, b}}=\frac{B(b)-B(a)}{b-a}
$$

where $a$ and $b$ indicate the lower and upper $\log \tau$ of compared maps, respectively.

Figure 8 (panels a and b) shows the maps of $(\Delta B / \Delta \log \tau)$ obtained by considering different atmospheric heights. Figure 8 (left panels) displays results by assuming $[a, b]=[\log \tau=$ $-0.5, \log \tau=-1.5]$ in the photosphere, and Figure 8 (right panels) displays results considering the heights $[a, b]=$ $[\log \tau=-1, \log \tau=-4.6]$ in the photosphere and chromosphere, respectively. Both the left- and right-hand panels display results for the penumbral regions labeled A (top panels) and B (middle panels) in Figures 2 and 5.

The inner part of the penumbra exhibits a ringlike structure in the $(\Delta B / \Delta \log \tau)_{-0.5,-1.5}$ map, clearly seen in Figure 8(a). In that ring structure, the photospheric vertical gradient of the field strength has negative values (i.e., the field strength decreases with the optical depth). Instead, the field gradient is positive in the outer part of the penumbra.

For the region labeled A, Figure 8 also displays the radial dependence of the vertical gradient of the magnetic field strength in the photosphere (panel c) and when moving from the photosphere to the chromosphere (panel d). We derived the radial dependence of the magnetic field by considering the field values at the locations of 80 isocontours in a smoothed map of the Fe I line-continuum intensity. This map was obtained by applying a boxcar running average values of intensity over $50 \times 50$ pixels. We then computed azimuthal averages of the field strength along the isocontours. We excluded from this analysis the B subarray because of the inhomogeneous penumbral filaments.

Figure 8(c) shows the radial dependence of the vertical gradient of the field strength $(\Delta B / \Delta \log \tau)_{-0.5,-1.5}$. At $r / R_{\text {spot }}=0.5$ in the inner penumbra, the field gradient is negative, with values of about $-100 \mathrm{G} / \log \tau$. From $r / R_{\text {spot }}=$ 0.55 , still in the inner penumbra, the field gradient starts to slightly increase. Moving further away from the umbra, at about $r / R_{\text {spot }}=0.6$, the value of the field gradient increases to mean values of about $300 \mathrm{G} / \log \tau$; from $r / R_{\text {spot }}=$ 0.6 to $r / R_{\text {spot }}=1.0$, the field gradient shows small fluctuations around the same mean value.

In contrast, the vertical gradient $(\Delta B / \Delta \log \tau)_{-1,-4.6}$ in Figure 8(d) displays positive values of about $200 \mathrm{G} / \log \tau$ at $r / R_{\text {spot }}=0.5$. The gradient slowly decreases when moving away from the umbra, assuming always positive values until $r / R_{\text {spot }}=1.0$. From $r / R_{\text {spot }}=0.5$ to $r / R_{\text {spot }}=0.6$ in the inner penumbra, the field gradient has an average value of about $170 \mathrm{G} / \log \tau$. From $r / R_{\text {spot }}=0.6$ onward, the gradient maintains to about $100 \mathrm{G} / \log \tau$. Overall, in the whole penumbra, the vertical gradient slightly decreases (i.e., the magnetic field increases with optical depth moving out from the sunspot center).

The above values of vertical magnetic field gradient are summarized in Table 2 .

\section{Discussion}

We analyzed the 3D structure of the magnetic field in the penumbra of a large and fully developed sunspot, as inferred from inversions of spectropolarimetric data taken along the $\mathrm{Fe}$ I $617.3 \mathrm{~nm}$ and Ca II $854.2 \mathrm{~nm}$ lines.

To verify the accuracy of our inversion results, we applied the NICOLE code to the Fe I and Ca II data separately, as recently done by other authors, such as Joshi \& de la Cruz Rodríguez (2018), and studied the results obtained with respect to those derived from the data inversion of both lines simultaneously. In this computational test, we assumed three nodes located from $\log \tau=-7$ and $\log \tau=1$ equidistantly for temperature, one node for each component of the vector magnetic field $\left(B_{x}, B_{y}\right.$, and $\left.B_{z}\right)$, one node for the LOS velocity, and one node for both the microturbulence and macroturbulence, respectively. This setting of the data inversion is similar to that used by Joshi \& de la Cruz Rodríguez (2018). Figure 9 shows the comparison of magnetic field strength values retrieved through the inversion of both the Fe I and $\mathrm{Ca}$ II lines 


\section{$\Delta \mathrm{B} / \Delta \log \tau[\mathrm{kG} / \log \tau]$}

$\begin{array}{lllll}-0.50 & -0.25 & 0.00 & 0.25 & 0.50\end{array}$

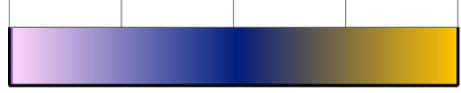

(a)
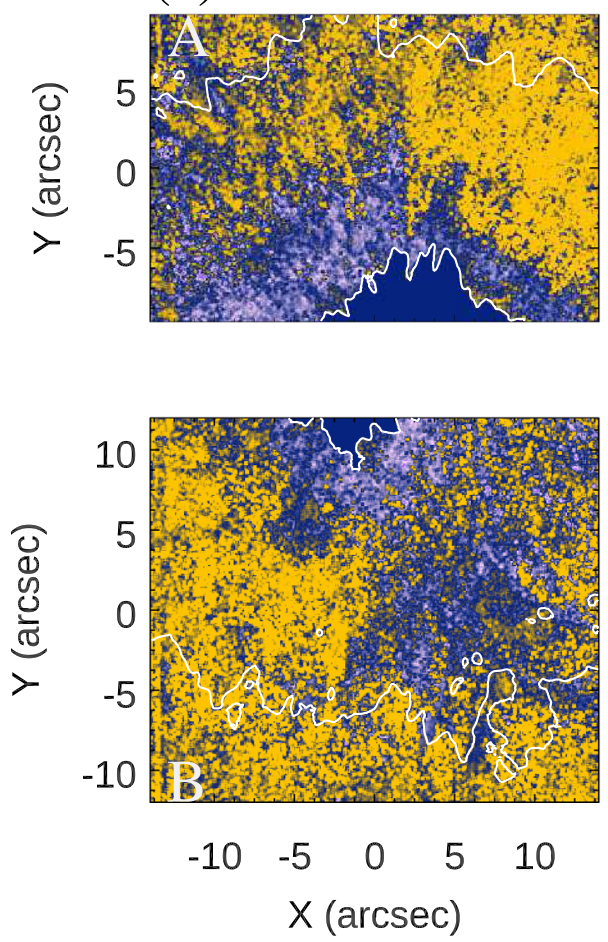

(c)

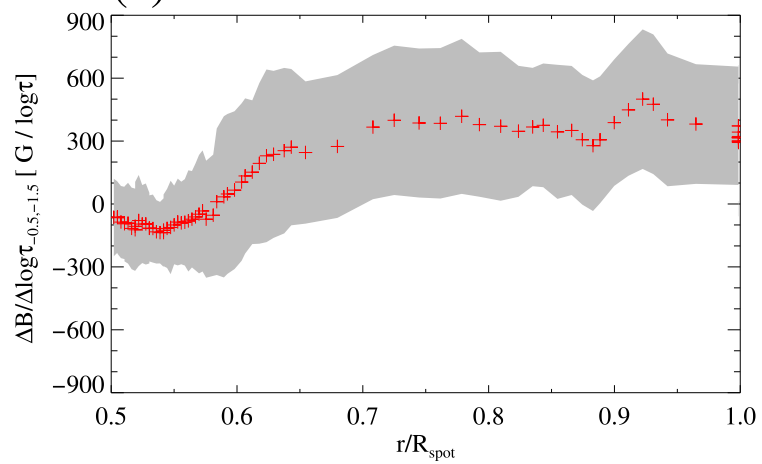

$\Delta \mathrm{B} / \Delta \log \tau[\mathrm{kG} / \log \tau]$

$\begin{array}{lllll}-0.2 & -0.1 & 0.0 & 0.1 & 0.2\end{array}$

(b)
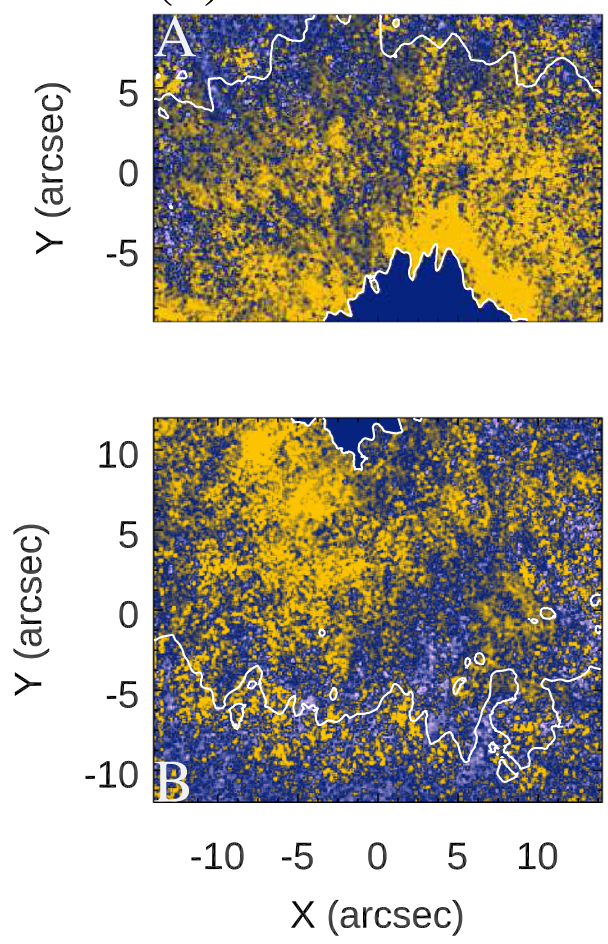

(d)

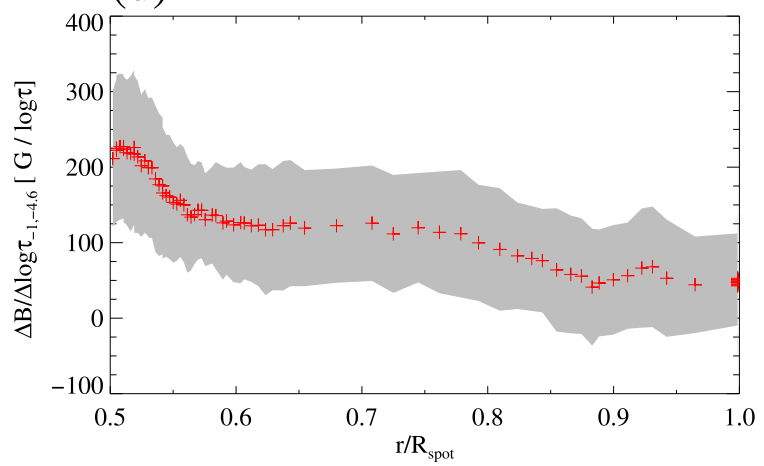

Figure 8. Panels (a) and (b) display the maps of the vertical gradient of the magnetic field strength by considering two atmospheric heights in the photosphere $(\Delta B / \Delta \log \tau)_{-0.5,-1.5}$ (left-hand panels) and two heights representative of the photosphere and chromosphere $(\Delta B / \Delta \log \tau)_{-1,-4.6}($ right-hand panels), for the subarrays A (top panels) and B (middle panels) shown in Figure 2 (panel b), respectively. White contours indicate the UP boundary and the outer boundary of the penumbra, as defined in Figure 2. For the subarray A, panels (c) and (d) show the variation of the vertical gradient of the magnetic field strength $(\Delta B / \Delta \log \tau)_{-0.5,-1.5}$ and $(\Delta B / \Delta \log \tau)_{-1,-4.6}$ as a function of $r / R_{\text {spot }}$, respectively. The shaded areas represent the standard deviation of the estimated field values.

data simultaneously and of the data of each line separately. This comparison is displayed at the optical depths where the RFs estimated as in Section 2.3 get maximum values, corresponding to $\log \tau=-1$ and $\log \tau=-4.6$ for the $\mathrm{Fe} \mathrm{I}$ and $\mathrm{Ca}$ II measurements, respectively. The red line in the panels represents one-to-one correspondence.

Figure 9 (left panel) displays that, at $\log \tau=-1$, the values of the magnetic field strength retrieved through the inversion of both lines simultaneously are in a close one-to-one correspondence to the values of the field obtained through the inversion of only the Fe I data. The relation between the compared series displays that fields whose strength ranges from 1.6 to $2.1 \mathrm{kG}$ have slightly lower values $(<2.5 \%)$ estimated by the simultaneous inversion of both lines with respect to findings from inversion of only Fe I data. At $\log \tau=-4.6$ (Figure 9, right panel), the correspondence between the values of the magnetic field strength retrieved through the inversion of both lines simultaneously are in reasonable agreement with those derived from the inversion of only the Ca II data, but the correspondence of values is less linear in comparison with the 

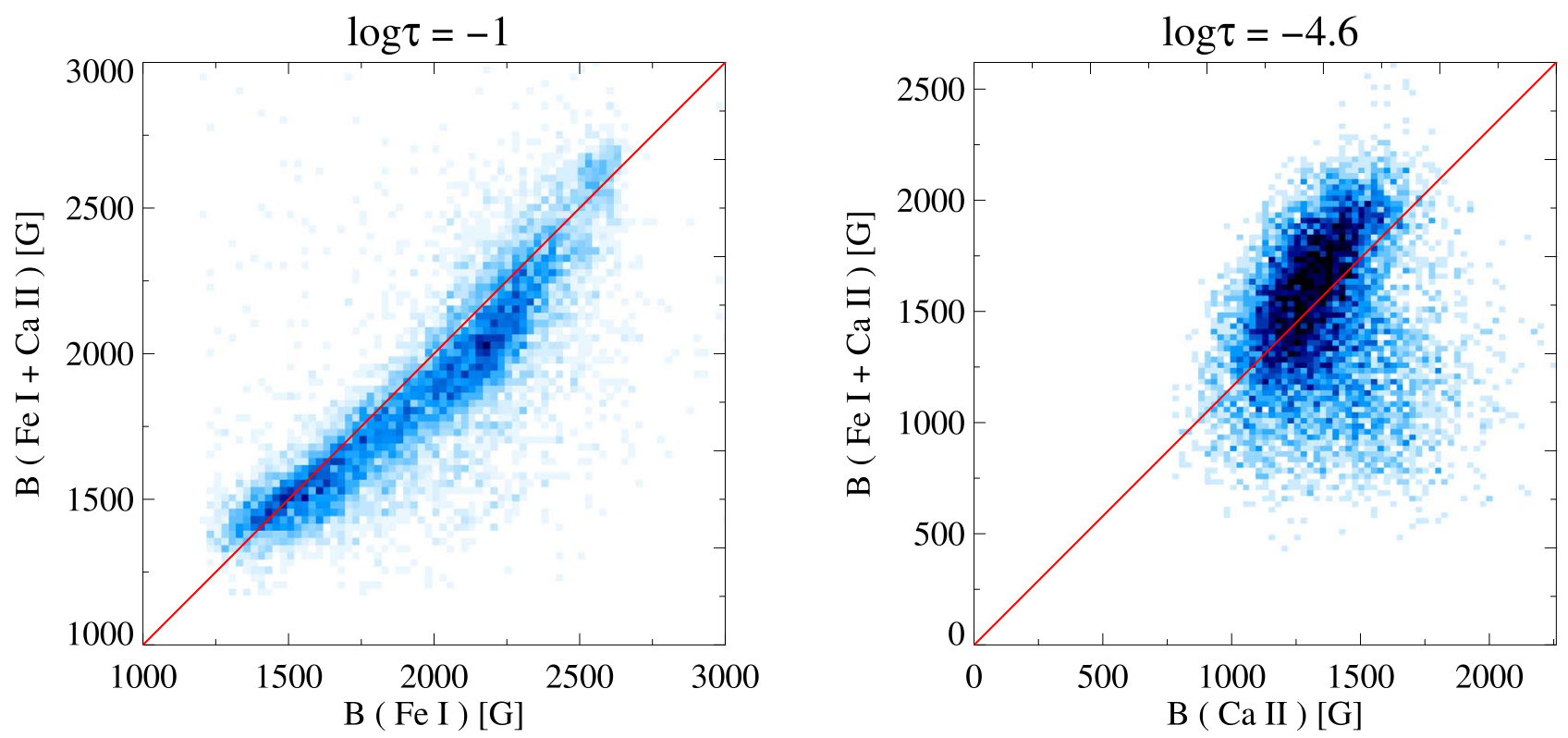

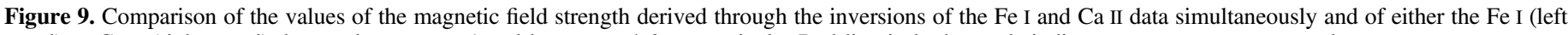
panel) or Ca II (right panel) data, at $\log \tau=-1$ and $\log \tau=-4.6$, respectively. Red line in both panels indicates one-to-one correspondence.

Table 2

Vertical Gradient of the Magnetic Field Strength

\begin{tabular}{lcc}
\hline \hline$r / R_{\text {spot }}$ & $\left(\frac{\Delta B}{\Delta \log \tau}\right)_{-0.5,-1.5}$ & $\left(\frac{\Delta B}{\Delta \log \tau}\right)_{-1,-4.6}$ \\
& $\mathrm{G} / \log / \log \tau$ \\
\hline$\leqslant 0.6$ & -110 & 170 \\
$>0.6$ and $\leqslant 0.9$ & 300 & 97 \\
$>0.9$ and $\leqslant 1.0$ & 350 & 46 \\
\hline
\end{tabular}

Note. $(\Delta B / \Delta \log \tau)_{-0.5,-1.5}$ : Mean value of the photospheric vertical gradient of the magnetic field strength between $\log \tau=-0.5$ and $\log \tau=-1.5$; $(\Delta B / \Delta \log \tau)_{-1,-4.6}$ : Mean value of the vertical gradient of the magnetic field strength between $\log \tau=-1.0$ and $\log \tau=-4.6$.

previous case. Moreover, for most of the image pixels, the simultaneous inversion of the data of both lines seems to produce slightly higher estimates of the field strength than that derived from inversion of the Ca II data only. However, the difference in results derived from the two inversion methods can be as high as $40 \%$ for the pixels characterized by large values of field strength. In addition, there are image pixels that exhibit lower field strengths (up to $30 \%$ ) with respect to those obtained from inversion of the $\mathrm{Ca}$ II line alone.

On the basis of these results, we consider that the stratification of the magnetic field obtained from our data inversion is reliable and accurate enough to discuss the findings from our study with respect to those in the literature. Furthermore, our findings derive from analysis of photospheric line data in addition to chromospheric Ca II line measurements. According to Quintero Noda et al. (2017), the method used in our study can greatly enhance the sensitivity of the analyzed data to the atmospheric parameters at lower heights.

We found that the magnetic field strength and inclination in the penumbra display a small-scale spine/intraspine structure at all of the atmospheric heights considered, from the photosphere to the middle chromosphere, though in the latter region, the small-scale pattern is attenuated with respect to the one observed deeper in the atmosphere.

The channellike structure of the vector magnetic field also weakens when moving along filaments away from the umbra. At the studied chromospheric height, we found signatures of the tails of penumbral filaments; in particular, at the outer border of the penumbra, we found patches of the horizontal magnetic fields with inclination of $\approx 90^{\circ}$ and field patches falling down to the photosphere with inclination of $\approx 105^{\circ}$.

Observations of the fine structure of penumbra in the chromosphere were recently presented by Joshi et al. (2016) on the basis of data acquired at the spectral region of the He I triplet with the $1.5 \mathrm{~m}$ class GREGOR telescope (Schmidt et al. 2012). Those authors found an azimuthal variation of the magnetic field inclination in the upper chromosphere, cospatial with the spine/intraspine pattern of inclination seen in the photosphere. The reported peak-to-peak variations of the inclination is about $\approx 10^{\circ}-15^{\circ}$ in the chromosphere, compared to the peak-to-peak variation of $\approx 20^{\circ}-25^{\circ}$ in the photosphere. Joshi et al. (2016) did not find any azimuthal variation of the magnetic field strength in the chromosphere, attributing this result to the higher magnetic pressure of plasma with respect to the gas pressure at atmospheric heights sampled by the He I triplet.

In contrast, the results derived from our study reveal a smallscale spine/intraspine structure of both the magnetic field strength and inclination in the middle chromosphere at the atmospheric heights sampled by the analyzed Ca II $854.2 \mathrm{~nm}$ data. Nevertheless, the small-scale field pattern is less structured when moving from the photosphere to the chromosphere; the fine-structuring of the field observed in the chromosphere coincides spatially well with that in the photosphere. We found typical peak-to-peak variations of the field strength and inclination of $\approx 300 \mathrm{G}$ and $\approx 20^{\circ}$, respectively, in the photosphere at $\log \tau=-0.5$, and of $\approx 200 \mathrm{G}$ and $\approx 10^{\circ}$ in the chromosphere at $\log \tau=-4.6$. The above values of the peak-to-peak variation of the magnetic field inclination are in agreement with those reported by Joshi et al. (2016). 
We also computed the photospheric vertical gradient $(\Delta B / \Delta \log \tau)_{-0.5,-1.5}$ in the northern and southern sectors of the studied penumbra. The maps of this quantity show a ringlike structure in the inner penumbra (from $r / R_{\text {spot }}=0.5$ to $\left.r / R_{\text {spot }} \approx 0.6\right)$ similar to the one reported by Joshi et al. (2017a) from analysis of photospheric data taken with the Tenerife Infrared Polarimeter-2 TIP-2 (Collados et al. 2007) mounted at the German Vacuum Tower Telescope (VTT) (between $\log \tau=-2.3$ and $\log \tau=0.0)$ and with the Hinode/SOT-SP (between $\log \tau=-0.9$ and $\log \tau=0.0$ ). However, our average photospheric $(\Delta B / \Delta \log \tau)$ is higher than that reported by Joshi et al. (2017a). In particular, we found values of the field gradient of about $300 \mathrm{G} / \log \tau$ when considering the photospheric heights $\log \tau=-0.5,-1.5$, whereas Joshi et al. (2017a) reported a value of about $50 \mathrm{G} / \log \tau$ considering data taken with the VTT/TIP-2 from $\log \tau=0.0$ and $\log \tau=-2.3$, and values of about $150 \mathrm{G} / \log \tau$ and $100 \mathrm{G} / \log \tau$ considering data taken with the Hinode/SOT-SP from $\log \tau=0.0$ and $\log \tau=-0.9$ and from $\log \tau=-0.9$ and $\log \tau=-2.5$, respectively. It is also worth nothing that our tests on field parameters estimated by the inversion of the data suggest that the field strength values derived from our study are, on average, smaller than those derived from inversion of photospheric data as in the previous studies. In addition, we computed the vertical gradient between the chromosphere and photosphere at the atmospheric heights where the observations analyzed in our study have maximum sensitivity to magnetic field variations. We found that the magnetic field strength decreases with optical depth in the penumbra. In particular, we report values of the field gradient of about $100 \mathrm{G} / \log \tau$ when comparing the field strength at photospheric and chromospheric heights $\log \tau=-1,-4.6$. By assuming the difference in the formation height of the Fe I $617.3 \mathrm{~nm}$ and the Ca II $854.2 \mathrm{~nm}$ lines derived from analysis of the phase difference in the propagation of the $p$-modes in the region studied by Stangalini et al. (2018), this difference is $\approx 300 \mathrm{~km}$ if the magnetoacoustic velocity is $7 \mathrm{~km} \mathrm{~s}^{-1}$, and the latter value of the vertical gradients of the magnetic field strength of $100 \mathrm{G} / \log \tau$ corresponds to a gradient of $\approx 0.3 \mathrm{G} \mathrm{km}^{-1}$. This result lies in the range of values reported by Joshi et al. (2017b), and much earlier by Rueedi et al. (1995), from analysis of chromospheric data taken in the spectral region of the He I $1083 \mathrm{~nm}$ triplet.

\section{Conclusions}

We studied the magnetic field strength and inclination in a sunspot penumbra, analyzing spectropolarimetric measurements at the Fe I $617.3 \mathrm{~nm}$ and $\mathrm{Ca}$ II $854.2 \mathrm{~nm}$ lines. The analyzed data sample the solar atmosphere from the deep photosphere to the middle chromosphere. To infer the physical properties of the penumbral plasma at different atmospheric heights, we inverted the available observations with the NICOLE code by processing both line measurements simultaneously. The results presented above derive from analysis of penumbral data acquired at spectral ranges unexplored in previous studies and inverted with different methods than those presented in the literature for similar investigations. In our maps of the magnetic field strength and inclination, the wellknown spine/intraspine structure is clearly seen at all of the atmospheric heights considered. In addition, we analyzed the vertical gradient of the magnetic field strength within different heights in the photosphere, as well as between heights in the photosphere and chromosphere, and found larger values for this quantity than reported from earlier studies. We draw attention to the fact that, on the basis of the values of the magnetic field strength estimated in the umbra, the gradient derived from our study may represent a peculiar case because of the extreme magnetic pressure reached in the umbra.

We presented results derived from inversion of photospheric and chromospheric observations, limiting the analysis to the observed regions where measured and inverted profiles are in good agreement. This is not the case for some observed umbral and UP boundary regions, which, however, were not the focus of the present work. Our study offers new observational constraints on the 3D magnetic structure of penumbral regions from analysis of data that sample chromospheric heights not considered in previous studies. However, it also clearly manifests the need for more data, as well as for simultaneous observations of penumbral regions by using multiple spectral diagnostics of the photosphere and chromosphere, to fully depict the 3D nature of the magnetic field at higher atmospheric heights. Indeed, these observing capabilities are foreseen to be available soon at the Swedish Solar Telescope, currently one of the most highly resolving solar telescopes, with the realization of the HeSp spectrometer working at the spectral range of the $\mathrm{He}$, in addition to the already operative CRisp Imaging SpectroPolarimeter (Scharmer 2006; Scharmer et al. 2008) CHROMospheric Imaging Spectrometer (CROMIS) working in the NUV-Vis bands (Scharmer 2017). These improved observing capabilities are also expected for the next-generation $4 \mathrm{~m}$ class solar telescopes, including the Daniel K. Inouye Solar Telescope (Keil et al. 2010) and European Solar Telescope (Collados et al. 2010), which are under construction and in the design phase, respectively.

The authors are grateful to the anonymous referee for constructive comments. The authors thank Doug Gilliam (NSO) and Michiel Van Noort (MPS) for their valuable support during the acquisition and MOMFBD processing of the data. S.L.G. and F.Z. are supported by Università degli Studi di Catania (Linea di intervento 1 e Linea di intervento 2). The research leading to these results has received funding from the European Research Council under the European Union's Horizon 2020 Framework Programme for Research and Innovation, grant agreements H2020 PRE-EST (No. 739500) and H2020 SOLARNET (No. 824135). This work was also supported by INAF Istituto Nazionale di Astrofisica (PRININAF-2014). S.J. acknowledges support from the European Research Council under the European Unions Horizon 2020 research and innovation program (grant agreement No. 682462) and from the Research Council of Norway through sits Centres of Excellence scheme (project No. 262622).

\section{ORCID iDs}

Mariarita Murabito (iD https://orcid.org/0000-0002-01442252

I. Ermolli (i) https://orcid.org/0000-0003-2596-9523 M. Stangalini (i) https://orcid.org/0000-0002-5365-7546

S. L. Guglielmino (i) https://orcid.org/0000-0002-1837-2262

S. Jafarzadeh (iD https://orcid.org/0000-0002-7711-5397

H. Socas-Navarro (i) https://orcid.org/0000-0001-9896-4622

P. Romano (iD https://orcid.org/0000-0001-7066-6674

F. Zuccarello (ib https://orcid.org/0000-0003-1853-2550 


\section{References}

Avrett, E. H., Fontenla, J. M., \& Loeser, R. 1994, in IAU. Symp. 154, Infrared Solar Physics, ed. D. M. Rabin, J. T. Jefferies, \& C. Lindsey (Dordrecht: Kluwer), 35

Balthasar, H. 2018, SoPh, 293, 120

Balthasar, H., \& Gömöry, P. 2008, A\&A, 488, 1085

Borrero, J. M., \& Ichimoto, K. 2011, LRSP, 8, 4

Cavallini, F. 2006, SoPh, 236, 415

Cauzzi, G., Reardon, K. P., Uitenbroek, H., et al. 2008, A\&A, 480, 515

Collados, M., Bettonvil, F., Cavaller, L. \& EST Team 2010, AN, 331, 615

Collados, M., Lagg, A., Díaz Garcí A, J. J., et al. 2007, in ASP Conf. Ser. 368, The Physics of Chromopheric Plasmas, ed. P. Heinzel, I. Dorotovič, \& R. J. Rutten (San Francisco, CA: ASP), 611

Felipe, T., Socas-Navarro, H., \& Przybylski, D. 2018, A\&A, 614, A73

Fontenla, J. M., Avrett, E. H., \& Loeser, R. 1993, ApJ, 406, 319

Guglielmino, S. L., Martínez Pillet, V., Bonet, J. A., et al. 2012, ApJ, 745, 160

Jafarzadeh, S., Rutten, R. J., Solanki, S. K., et al. 2017, ApJS, 229, 11

Joshi, J., \& de la Cruz Rodríguez, J. 2018, A\&A, 619, A63

Joshi, J., Lagg, A., Hirzberger, J., \& Solanki, S. K. 2017b, A\&A, 604, A98

Joshi, J., Lagg, A., Hirzberger, J., Solanki, S. K., \& Tiwari, S. K. 2017a, A\&A, 599, A35

Joshi, J., Lagg, A., Solanki, S. K., et al. 2016, A\&A, 596, A8

Keil, S. L., Rimmele, T. R., Wagner, J. \& ATST Team 2010, AN, 331, 609

Lagg, A., Lites, B., Harvey, J., Gosain, S., \& Centeno, R. 2017, SSRv, 210, 37

Lites, B. W., Akin, D. L., Card, G., et al. 2013, SoPh, 283, 579

Livingston, W., \& Watson, F. 2015, GeoRL, 42, 9185

Loughhead, R. E. 1968, SoPh, 5, 489

Mathew, S. K., Lagg, A., Solanki, S. K., et al. 2003, A\&A, 410, 695

Murabito, M., Romano, P., Guglielmino, S. L., \& Zuccarello, F. 2017, ApJ, 834,76

Murabito, M., Romano, P., Guglielmino, S. L., Zuccarello, F., \& Solanki, S. K. 2016, ApJ, 825, 75

Murabito, M., Zuccarello, F., Guglielmino, S. L., \& Romano, P. 2018, ApJ, 855,58

Orozco Suarez, D., Asensio Ramos, A., \& Trujillo Bueno, J. 2015, ApJL, 803, L18

Pesnell, W. D., Thompson, B. J., \& Chamberlin, P. C. 2012, SoPh, 275, 3

Quintero Noda, C., Shimizu, T., de la Cruz Rodríguez, J., et al. 2016, MNRAS, 459,3363

Quintero Noda, C., Shimizu, T., Katsukawa, Y., et al. 2017, MNRAS, 464, 4534
Reardon, K. R., \& Cavallini, F. 2008, A\&A, 481, 897

Rempel, M., \& Schlichenmaier, R. 2011, LRSP, 8, 3

Rimmele, T. R. 2004, ApJ, 604, 906

Robustini, C., Leenaarts, J., \& de la Cruz Rodríguez, J. 2018, A\&A, 609, A14

Romano, P., Frasca, D., Guglielmino, S. L., et al. 2013, ApJL, 771, L3

Romano, P., Guglielmino, S. L., Cristaldi, A., et al. 2014, ApJ, 784, A10

Rueedi, I., Solanki, S. K., \& Livingston, W. 1995, A\&A, 302, 543

Sánchez Cuberes, M., Puschmann, K. G., \& Wiehr, E. 2005, A\&A, 440, 345

Scharmer, G. B. 2006, A\&A, 447, 1111

Scharmer, G. B. 2017, in SOLARNET IV: The Physics of the Sun from the Interior to the Outer Atmosphere (Tenerife, Spain: Instituto de Astrofísica de Canarias), 85

Scharmer, G. B., Narayan, G., Hillberg, T., et al. 2008, ApJL, 689, L69

Scherrer, P. H., Schou, J., Bush, R. I., et al. 2012, SoPh, 275, 207

Schlichenmaier, R., Rezaei, R., \& Bello González, N. 2012, in ASP Conf. Ser 455, IV Hinode Science Meeting: Unsolved Problems and Recent Insights,, ed. L. Bellot Rubio, F. Reale, \& M. Carlsson (San Francisco, CA: ASP), 61

Schlichenmaier, R., Rezaei, R., Bello González, N., \& Waldmann, T. A. 2010, A\&A, 512, L1

Schmidt, W., von der Luhe, O., Volkmer, R., et al. 2012, AN, 333, 796

Socas-Navarro, H. 2005a, ApJL, 631, L167

Socas-Navarro, H. 2005b, ApJL, 633, L57

Socas-Navarro, H., de la Cruz Rodríguez, J., Asensio Ramos, A., Trujillo Bueno, J., \& Ruiz Cobo, B. 2015, A\&A, 577, A7

Solanki, S. K. 2003, A\&ARv, 11, 153

Solanki, S. K., \& Montavon, C. A. P. 1993, A\&A, 275, 283

Stangalini, M., Jafarzadeh, S., Ermolli, I., et al. 2018, ApJ, 869, 110

Thomas, J. H., Weiss, N. O., Tobias, S. M., \& Brummell, N. H. 2002, Natur, 420, 390

Tiwari, S. K. \& Hinode Review Team 2017, PASJ, submitted (arXiv:1712. 07174)

Tiwari, S. K., van Noort, M., Lagg, A., \& Solanki, S. K. 2013, A\&A, 557, A25

Tiwari, S. K., van Noort, M., Solanki, S. K., \& Lagg, A. 2015, A\&A, 583, A119

Tsuneta, S., Ichimoto, K., Katsukawa, Y., et al. 2008, SoPh, 249, 167

van Driel-Gesztelyi, L., \& Green, L. M. 2015, LRSP, 12, 1

van Noort, M., Rouppe van der Voort, L., \& Löfdahl, M. G. 2005, SoPh, 228, 191

Westendorp Plaza, C., del Toro Iniesta, J. C., Ruiz Cobo, B., et al. 2001, ApJ, 547,1130 